\title{
Comparison between "early" or "late" intravitreal injection of dexamethasone implant in branch (BRVO) or central (CRVO) retinal vein occlusion: six months follow-up
}

Fernanda Pacella, Giuseppe La Torre, Stefania Basili, Monica Autolitano, Antonella Pascarella, Tommaso Lenzi \& Elena Pacella

To cite this article: Fernanda Pacella, Giuseppe La Torre, Stefania Basili, Monica Autolitano, Antonella Pascarella, Tommaso Lenzi \& Elena Pacella (2016): Comparison between "early" or "late" intravitreal injection of dexamethasone implant in branch (BRVO) or central (CRVO) retinal vein occlusion: six months follow-up, Cutaneous and Ocular Toxicology, DOI: $\underline{10.1080 / 15569527.2016 .1254648}$

To link to this article: http://dx.doi.org/10.1080/15569527.2016.1254648

Accepted author version posted online: 30

Nov 2016.

Submit your article to this journal [

View related articles ¿

View Crossmark data \lceil 


\title{
Comparison between "early" or "late" intravitreal injection of dexamethasone implant in branch (BRVO) or central (CRVO) retinal vein occlusion: six months follow-up
}

\begin{abstract}
Purpose: The purpose of this study was to compare early and late injections of intravitreal dexamethasone implant in patients affected by central retinal vein occlusion (CRVO) or branch retinal vein occlusion (BRVO) with a six-month follow-up. We assessed whether an earlier treatment start (within 7 days from diagnosis) could be more beneficial than a delayed (or late) treatment start (after 7 days).
\end{abstract}

Materials and Methods: The study included 81 patients ( 81 eyes) affected by retinal vein occlusion. Best corrected visual acuity was assessed through Early Treatment Diabetic Retinopathy Study (ETDRS) while central macular thickness (CMT) was measured by spectral-domain optical coherence tomography.

Results: Both types of patients had a positive therapeutic response to dexamethasone, with an increase in visual acuity (ETDRS) and CMT reduction. CRVO patients were characterized by lower ETDRS values at baseline and at the end of the follow-up as compared to BRVO. CRVO patients showed higher CMT values at baseline, after 3 and six months from injection. No significant differences in therapeutic response to dexamethasone were observed between patients treated early or late, regardless of RVO type.

Conclusions: This study demonstrates that the therapeutic properties of dexamethasone implant are not significantly influenced by an early or late treatment start in patients affected by BRVO and CRVO, although its therapeutic efficacy seems greater in the former type.

Key words: Retinal vein occlusion; dexamethasone implant; intravitreal, BRVO; CRVO 


\section{Introduction}

Retinal vein occlusion (RVO) is a common vascular disorder of the retina involving either the central or branch retinal veins. RVO represents the second most common cause of vision loss from retinal vascular disease, following diabetic retinopathy (1). RVO causes an increase in retinal capillary pressure, which results in up-regulation of vascular endothelial growth factor (VEGF) expression and a consequent increase in vascular permeability (2). These effects trigger complications, including macular edema and varying degrees of ischemia, and potentially lead to severe vision loss. The prognosis for branch RVO (BRVO) is frequently more favorable than that for central RVO (CRVO) (3).

At present, anti-VEGF drugs have revolutionized the clinical course and prognosis of patients with RVOs (4). The anti-VEGF agents have been recognized as the first-line treatment for macular edema in both types of RVO. Anti-VEGF drugs such as Ranibizumab (Lucentis®, Genentech Inc., San Francisco, California, USA and Novartis Pharma AG, Basel, Switzerland), Aflibercept (EYLEA; Bayer HealthCare, Berlin, Germany), and bevacizumab (Avastin; Genentech Inc.) and Bevacizumab (Avastin, Genentech) have been widely used by ophthalmologists to treat RVO (5). Although Ranibizumab has been licensed for the treatment of patients with macular edema secondary to RVO (6), in 2005 the off-label use of bevacizumab for RVO treatment was introduced because of its similar target specificity and lower cost (7). Despite this, there is lack of large-scale clinical trial data supporting the use of bevacizumab (5), and single cases of complications associated with its utilization have been reported (8).

More recently, the use of intravitreal corticosteroids, such as dexamethasone, triamcinolone acetonide and fluocinolone acetonide have been also introduced for the treatment of RVO (9-14). Corticosteroids including dexamethasone are known to have anti-inflammatory, anti-angiogenic properties and may inhibit the expression of VEGF, the adhesion molecule ICAM-1, proinflammatory cytokines (i.e. interleukin-6) and chemokines (MCP-1) (15-17). 
In 2009, a sustained-release intravitreal $0.7 \mathrm{mg}$ dexamethasone delivery system, Ozurdex (Allergan Inc., Irvine, CA, USA), was approved for treatment of macular edema secondary to RVO. Dexamethasone intravitreal implant is a biodegradable dexamethasone-releasing implant containing the corticosteroid dexamethasone. The implant is applied 3-4 $\mathrm{mm}$ posterior to the limbus, does not require surgical removal, and if necessary, it allows you to place another implant. Two phases of drug release are observed. The first phase is characterized by 2-month high concentration of the drug release. Successively, drug release decreases over time and within 6-month period drug concentration declines to non quantifiable levels (18).

Ozurdex has demonstrated efficacy and safety for the treatment of BRVO and CRVO (12) and was also approved for the management of diabetes, noninfectious posterior uveitis and Irvine-Gass syndrome. In addition, intravitreal dexamethasone has shown to have better therapeutic effects in patients affected by diabetic macular edema previously treated with other corticosteroids such as intravitreal triamcinolone (19)

Previous studies on RVO disease course have shown that a macular edema is present from the beginning and its persistence is associated with poor visual prognosis (20-22). In addition, the GENEVA study showed greater improvement of symptoms in the group of patients with shorter duration of macular edema (12). Thus, there is the possibility that an earlier treatment start could give better outcome in anatomical and functional results as compared to a delayed treatment start.

To test this hypothesis in this study we compared the effect of intravitreal injection of dexamethasone implant in BRVO and CRVO patients who received an earlier (within 7 days from diagnosis) or a delayed treatment start (after 7 days from diagnosis). In these patients, we measured the best corrected visual acuity (BCVA) and central macular thickness (CMT) at baseline and after 1,3 , and 6 months post injection. 


\section{Materials and methods}

\section{Patient selection}

The subjects were recruited at Department of Sense Organs, Faculty of Medicine and Dentistry, Sapienza University of Rome, Italy. Informed consent was obtained from all subjects. The study adhered to the tenets of the Declaration of Helsinki for research involving human subjects.

The study included 81 patients (81 eyes; 53 males and 28 females) all affected by retinal vein occlusion (Table 1). Mean age was $62.5 \pm 15.3$ years. All eyes affected were treated with Ozurdex ${ }^{\circledR}$. The timing to injection was recorded since start of symptoms, after the diagnosis of RVO.

[Table 1 near here]

\section{Inclusion criteria}

Criteria for inclusion were: (a) age >18 years old; (b) macular edema secondary to central or branch retinal vein occlusion; (c) BCVA measured by ETDRS <45 letters and between 0.2 and 1.0 LogMAR at baseline examination; (d) CMT $\geq 285 \mu \mathrm{m}$ measured by spectral-domain optical coherence tomography (SD-OCT) at baseline examination; (e) intraocular pressure under 21 $\mathrm{mmHg}$; (f) subjects free of treatment (intravitreal or laser) for their vein occlusion.

\section{Exclusion criteria}

The exclusion criteria were: (a) previous laser treatment or intravitreal injection; (b) uncontrolled arterial hypertension; (c) subjects with high-grade cataracts; (d) glaucoma; (e) epiretinal membrane involving fovea or organized hard exudative plaques; (f) age-related macular degeneration (AMD);(g) any ocular surgery in the study eye in the last six months; (h) pregnancy; (i) case-history for intraocular pressure (IOP) elevation in response to steroid treatment; (j) uveitis. 


\section{Efficacy criteria}

- Primary outcome measure was the changes in mean BCVA values before and after the treatment. The implant was considered efficient when a mean improvement of BCVA $\geq 10$ letters (2 lines ETDRS) was observed.;

- Secondary outcome measure was the changes in CMT values between baseline and follow-up visits trough the analysis of the retinal layer structure using OCT. The expected outcome was a reduced mean $\mathrm{CMT} \geq 250 \mu \mathrm{m}$. The evaluation of the integrity of the external membrane and of the inner and outer segments of the photoreceptor interface was carried out at baseline (T0) and after 1 (T1), 3 (T3), and 6 months (T6) post injection.

\section{Safety Criteria}

The insurgence of undesired side-effects correlated to the drug (i.e. inflammation of the anterior chamber; ocular pain; keratitis or vitreous opacity; increase in intraocular pressure and insurgence of cataract) was monitored monthly.

Side effects correlated to the intravitreal therapy (i.e. endophthalmitis; perforation of the eye; conjunctival hemorrhage and systemic effects related to the drug) were also monitored monthly.

\section{Procedure of Intravitreal Dexamethasone Implant}

All implants were performed under sterile conditions. Povidone-iodine solution (5\%) and topical anesthetic ( ropivacaine) were used to prepare the conjunctiva and the blepharostat was positioned. A 700 microgram slow release Intravitreal Dexamethasone Implant (Ozurdex $\left.{ }^{\circledR}\right)$ was placed in the vitreal cavity behind the crystalline lens within $3 \pm 2$ days from baseline examination. All injections were performed in an operating room and the dexamethasone implant was inserted into the vitreous cavity through the pars plana using a customized single-use 22-gauge applicator (see ref. 16 for further details). 


\section{Evaluations}

Baseline evaluation of vision carried out pre-therapy at T0 included: fluorangiography performed to evaluate the presence of macular ischemia, BCVA assessed through ETDRS tables placed at a distance of $4 \mathrm{~m}$ by slit lamp biomicroscopy, ocular tonometry (using a Goldman applanation tonometer), fundus biomicroscopy, optical coherence tomography (OCT) (for measurement of macular thickness and morphology using a Spectralis HRA-OCT produced by Heidelberg Engineering with a volumetric 512 x 49-scan) and color fundus photography. CMT was measured by spectral-domain optical coherence tomography.

BCVA and CMT exams were carried out at baseline (T0) and repeated after 1 (T1), 3 (T3), and 6 months (T6) post injection.

\section{Study groups}

To evaluate the difference in treatment response between the RVO types, patients were divided in two groups according to their clinical records: (a) patients affected by CRVO (n=46) and (b) patients affected by BRVO $(n=35)$. A sham treatment group, although helpful to improve the quality of the study, was not included for ethical reasons.

Successively, to evaluate the differences between an early and late treatment start, patients were also divided in the following two groups: (c) patients where the treatment was administered within 7 days from the initial RVO diagnosis $(\leq 7 \mathrm{~d})(\mathrm{n}=38 ; 22$ BRVO/16 CRVO) and (d) patients where the treatment was administered later than 7 days from RVO diagnosis $(>7 \mathrm{~d})(\mathrm{n}=43 ; 13 \mathrm{BRVO} / 30$ CRVO).

The choice to compare patients injected before and after 7 days was not based on previous clinical data but on examination of clinical records at admission to our ophthalmic emergency unit. Although all patients were immediately treated with Ozurdex, some of them were diagnosed in a very initial phase of RVO at admission while others in a more advanced phase of disease with a time window of 8-30 days. 


\section{Statistical analysis}

Data were analyzed by ANOVA with repeated measures with diagnosis (BRVO/CRVO), treatment time (within/after 7 days from diagnosis) and time (months from injection) as variables. Post-hoc comparisons were performed with Fisher's Protected Least Significant Difference (PLSD) post-hoc test. A p-value $<0.05$ was considered statistically significant. Statistical analysis was performed using the Statview software from SAS Institute.

\section{Results}

Visual acuity measured with ETDRS Scale after intravitreal dexamethasone implant in BRVO and CRVO patients

Repeated measures ANOVA showed that intravitreal dexamethasone implant had a significant effect over treatment time on visual acuity $(\mathrm{p}<0.001)$ (Fig. 1). Post-hoc analysis showed that ETDRS values were elevated at T1 $(\mathrm{p}<0.001), \mathrm{T} 3(\mathrm{p}<0.001)$ and $\mathrm{T} 6(\mathrm{p}<0.001)$ as compared to T0 in both BRVO and CRVO patients. We also observed that patients affected by BRVO had increased ETDRS values at baseline $(\mathrm{p}<0.05)$ and T6 $(\mathrm{p}<0.05)$ as compared to CRVO patients (Fig.1) (Table 2).

[Figure 1 near here]

[Table 2 near here] 
ETDRS values in patients treated before or after 7 days from diagnosis

The differences in ETDRS values between patients treated before or after 7 days from diagnosis are shown in Fig. 2. ANOVA did not show significant differences between the two groups. We observed a significant effect of intravitreal dexamethasone treatment $(\mathrm{p}<0.05)$. Post-hoc analysis showed that dexamethasone increased ETDRS values at T1 $(\mathrm{p}<0.001), \mathrm{T} 3(\mathrm{p}<0.001)$ and T6 $(\mathrm{p}<0.001)$ as compared to T0.

[Figure 2 near here]

\section{CMT after intravitreal dexamethasone implant in BRVO and CRVO patients}

Repeated measures ANOVA showed that intravitreal dexamethasone implant had a significant effect over treatment time on CMT (p<0.001) (Fig. 3). Post-hoc analysis showed that CMT values were reduced at T1 $(\mathrm{p}<0.001), \mathrm{T} 3(\mathrm{p}<0.001)$ and T6 $(\mathrm{p}<0.001)$ as compared to T0 in both BRVO and CRVO patients.

Patients affected by BRVO had decreased CMT values at baseline $(\mathrm{p}<0.05)$, T3 $(\mathrm{p}<0.05)$ and T6 $(\mathrm{p}<0.05)$ as compared to CRVO patients (Table 2).

[Figure 3 near here]

\section{CMT values in patients treated before or after 7 days from diagnosis}

The differences in CMT values between patients treated before or after 7 days from diagnosis are shown in Fig. 4. ANOVA did not show significant differences between the two groups. We observed a significant effect of intravitreal dexamethasone treatment $(\mathrm{p}<0.001)$. Post-hoc analysis showed that dexamethasone significantly reduced CMT values at T1 $(\mathrm{p}<0.001), \mathrm{T} 3(\mathrm{p}<0.001)$ and T6 (p<0.001) as compared to T0 (Fig. 4) (Table 2). 
[Figure 4 near here]

\section{Side effects}

Intraocular pressure (IOP) of each patient did not shown significant increment during the time of the study and in the successive follow-up. None of the patients had uncontrolled hypertension, hyperlipidemia, renal disease, or other comorbidities at the time of inclusion or during the follow-up period. In 5 patients where IOP increased over $21 \mathrm{mmHg}$, this condition was successfully treated with beta blockers.

Six patients had a worsening of their cataract during the follow-up period, presumably due to dexamethasone adverse reaction. None of the patients showed side effects correlated to the surgical intervention itself.

\section{Discussion}

This study was performed to investigate whether the therapeutic effect of dexamethasone intravitreal implants in patients affected by branch (BRVO) or central (CRVO) retinal vein occlusion may depend on the interval between diagnosis and treatment start. This idea was generated by data reported in the GENEVA study showing that the group of patients with shorter duration of macular edema were characterized by greater symptom improvement (12). Therefore, in this study we aimed at evaluating whether an earlier treatment start (within 7 days from diagnosis) could be more beneficial than a delayed treatment start (after 7 days from diagnosis).

The results showed that both types of patients had a positive therapeutic response to dexamethasone, characterized by an increase in visual acuity (ETDRS) and reduction of CMT. CRVO patients were characterized by lower ETDRS values at baseline and at the end of the follow- 
up as compared to BRVO. CRVO patients also showed greater CMT values at baseline, after three and six months from injection. No significant differences on therapeutic response to dexamethasone were observed between patients treated early or late, regardless of RVO type.

The main finding of our study is that the interval between diagnosis and treatment has not significant effects on the therapeutic response to dexamethasone implant in BRVO and CRVO patients. These data suggest that the response to dexamethasone is not influenced by the time elapsed between diagnosis and treatment, and that the disease process could be reversed in an acceptable time window.

Although the optimal treatment strategy for RVO is still matter of debate, these data suggest that dexamethasone implant is a good therapeutic option for the treatment of RVO. Natarajan (23) states that, in the majority of cases of BRVO, the disease may undergo spontaneous regression after 3 months. However, this hypothesis is not always confirmed in the clinical practice. In such cases, anti-angiogenic drugs, like ranibizumab, pegaptanib and bevacizumab, represent the best treatment options without causing tissue damage (24-26). In particular, antiangiogenic drugs may be useful for reducing both edema and neo-vascularization in occlusive pathologies $(24,27,28)$.

Dexamethasone implants showed similar therapeutic profile in both BRVO and CRVO patients. These data are in line with previous reports demonstrating the efficacy of dexamethasone for the treatment of macular edema secondary to retinal vein occlusion (29). As reported in a previous study (30), its therapeutic effect was more evident for BRVO cases, although it should be noted that BRVO patients, at least in our cohort, were characterized by better condition at baseline as compared to CRVO. BRVO has generally a more favorable prognosis than that of CRVO $(21,31)$. This could be explained by the fact that, although the exact pathogenesis of RVO remains unclear, CRVO and BRVO are different entities with different prognosis and management (32).

In any case, this study shows that dexamethasone implant could be beneficial in both types, without significant side effects. This latter finding is in line with the randomized GENEVA clinical trial (12), which was also sham-controlled, performed to evaluate the safety and efficacy of two doses of 
dexamethasone intravitreal implant $(0.7$ and $0.35 \mathrm{mg})$ in patients affected by macular edema associated with BRVO or CRVO. That study demonstrated that, after a single injection, both groups of patients had a significant letter improvement in BCVA $(>15)$ as compared to the sham group, without significant differences in the rate of complications, such as cataract or increased IOP. On the opposite, another clinical trial, the SCORE study (33), performed with another corticosteroid administered intravitreally, triamcinolone, did not find differences in visual acuity and CMT in a group of BRVO patients as compared to the group treated with grid photocoagulation. The conclusion was that, although the two treatments had a similar outcome on visual acuity, the rates of adverse events (particularly elevated IOP and cataract) were highest in the triamcinolone (4-mg) group. The reason for such discrepancy are not clear. It is possible that the two corticosteroids may have different effects on patients depending on their composition, preparation and dosages (34). Interestingly, a later analysis of the GENEVA and SCORE studies (35) reported that those studies provide insufficient evidence to support the use of intravitreal steroids in CRVO patients, because of a high risk of bias due to incomplete outcome data in the SCORE study and selective outcome reporting in the GENEVA study. It should be also noted that the lower rates of these adverse events (increased IOP and cataract) in our cohort could be due to more restrictive "inclusion and/or exclusion criteria" as compared to other studies, possibly leading to the formation of a healthier study group. It is possible that our data on IOP elevation are different from those of the GENEVA and other studies because of the timing of measurements. We found indeed that after four weeks IOP values were constant in CRVO and BRVO patients as compared to baseline measurements. In addition, patients presenting IOP elevation were treated with local antihypertonic drop, a fact that may have contributed to reduce steroid-induced IOP elevation. In fact after 12 weeks IOP in these patients was under $21 \mathrm{mmHg}$. It is unlikely that differences in clinical evaluation and/or intravitreal procedure may have resulted in lower rate of complication. Thus, these studies together with ours indicate the use of corticosteroid intravitreal implants probably needs to be further developed to 
avoid the possibility that implants with prolonged release characteristics may increase the risk for ocular side effects such as cataract and glaucoma.

There are some limitation to the interpretation of our findings. A six-month follow-up is a relatively short period to reach definitive conclusions. Thus, these data need to be confirmed in successive studies with larger cohorts of patients and for a longer period of time. Moreover, it is desirable that future trials will compare different treatment strategies (i.e. dexamethasone vs anti-VEGF) or combination therapies within the same study and evaluate the efficacy of repeat treatments for continued symptom relief.

In conclusion, this study shows that the therapeutic response to dexamethasone implant in patients affected by BRVO and CRVO may not be influenced by the time elapsed between diagnosis and treatment, although its therapeutic efficacy seems greater in BRVO patients.

\section{Acknowledgements}

None

\section{Disclosure of interest}

The authors report no conflicts of interest. 


\section{References}

1. Cugati S, Wang JJ, Rochtchina E, Mitchell P. Ten-year incidence of retinal vein occlusion in an older population: the Blue Mountains Eye Study. Arch Ophthalmol. 2006;124:726-32.

2. Miller JW, Le Couter J, Strauss EC, Ferrara N. Vascular endothelial growth factor a in intraocular vascular disease. Ophthalmology 2013;120:106-14.

3. Eye Disease Case-Control Study Group. Risk factors for branch retinal vein occlusion. Am J Ophthalmol. 1993;116:286-96.

4. Ashraf M, Souka AA, Singh RP. Central retinal vein occlusion: modifying current treatment protocols. Eye (Lond). 2016 Feb 12. Doi: 10.1038/eye.2016.10. [Epub ahead of print] Review.

5. Campa C, Alivernini G, Bolletta E, Parodi MB, Perri P. Anti-VEGF therapy for retinal vein occlusions. Curr Drug Targets 2016;17:328-36.

6. Song WT, Xia XB. Ranibizumab for macular edema secondary to retinal vein occlusion: a meta-analysis of dose effects and comparison with no anti-VEGF treatment. BMC Ophthalmol. 2015;15:31.

7. Rosenfeld PJ, Fung AE, Puliafito CA. Optical coherence tomography findings after an intravitreal injection of bevacizumab (avastin) for macular edema from central retinal vein occlusion. Ophthalmic Surg Lasers Imaging 2005;36:336-9.

8. Pacella, E, Turchetti P, Artico M, Piraino CD, Mollicone A, Bottone A, et al. Adverse Events Associated with Intraocular Injection of Anti-VEGF(bevacizumab) in Retinal Vein Occlusion: A Case Report. Neuro-Ophthalmology \& Visual Neurosci. 2015;1:22-6.

9. Thom HH, Capkun G, Nixon RM, Ferreira A. Indirect comparisons of ranibizumab and dexamethasone in macular oedema secondary to retinal vein occlusion. BMC Med Res Methodol. 2014;14:140. 
10. Nghiem-Buffet S, Fajnkuchen F, Buffet M, Ayrault S, Le Gloahec-Lorcy A, Grenet T, et al. Intravitrealranibizumab and/or dexamethasone implant for macular edema secondary to retinal vein occlusion. Ophthalmologica 2014;232:216-22.

11. Glanville J, Patterson J, McCool R, Ferreira A, Gairy K, Pearce I. Efficacy and safety of widely used treatments for macular oedema secondary to retinal vein occlusion: a systematic review. BMC Ophthalmol. 2014;14:7.

12. Haller JA, Bandello F, Belfort R Jr, Blumenkranz MS, Gillies M, Heier J, et al. Randomized, sham-controlled trial of dexamethasone intravitreal implant in patients with macular edema due to retinal vein occlusion. Ophthalmology 2010;117:1134-46. E1133.

13. Jonas JB, Kreissig I, Degenring RF. Intravitreal triamcinolone acetonide as treatment of macular edema in central retinal vein occlusion. Graefes Arch Clin Exp Ophthalmol. 2002;240:782-783.

14. Jain N, Stinnett SS, Jaffe GJ. Prospective study of a fluocinolone acetonide implant for chronic macular edema from central retinal vein occlusion: thirty-six-month results. Ophthalmology. 2012;119:132-137.

15. Koss MJ, Pfister M, Rothweiler F, Michaelis M, Cinatl J, Schubert R, et al. Comparison of cytokine levels from undiluted vitreous of untreated patients with retinal vein occlusion. Acta Ophthalmol. 2012;90:e98-e103.

16. Wang K, Wang Y, Gao L, Li X, Li M, Guo J. Dexamethasone inhibits leukocyte accumulation and vascular permeability in retina of streptozotocin-induced diabetic rats via reducing vascular endothelial growth factor and intercellular adhesion molecule-1 expression. Biol Pharm Bull. $2008 ; 31: 1541-6$.

17. Pfister M, Rothweiler F, Michaelis M, Cinatl J Jr, Schubert R, Koch FH, et al. Correlation of inflammatory and proangiogenic cytokines from undiluted vitreous samples with spectral domain OCT scans, in untreated branch retinal vein occlusion. Clin Ophthalmol. 2013;7:10617. 
18. Chang-Lin JE, Attar M, Acheampong AA, Robinson MR, Whitcup SM, Kuppermann BD, Welty D. Pharmacokinetics and pharmacodynamics of a sustained-release dexamethasone intravitreal implant. Invest Ophthalmol Vis Sci. 2011;52(1):80-6.

19. Augustin AJ, Kuppermann BD, Lanzetta P, Loewenstein A, Li XY, Cui H, Hashad Y, Whitcup SM; Ozurdex MEAD Study Group. Dexamethasone intravitreal implant in previously treated patients with diabetic macular edema: subgroup analysis of the MEAD study. BMC Ophthalmol. 2015;15:150.

20. Central Retinal Vein Occlusion Study Group: Natural history and clinical management of central retinal vein occlusion. Arch Ophthalmol 1997; 115: 486-491.

21. McIntosh RL, Rogers SL, Lim L, Cheung N, Wang JJ, Mitchell P, et al. Natural history of central retinal vein occlusion: an evidence-based systematic review. Ophthalmology 2010;117:1113-23. E1115.

22.. Pacella E, Vestri AR, Muscella R, Carbotti MR, Castellucci M, Coi L, et al. Preliminary results of an intravitreal dexamethasone implant $($ Ozurdex $®)$ in patients with persistent diabetic macular edema. Clin Ophthalmol. 2013;7:1423-8.

23. Natarajan S. Managing patients with retinal vein occlusions: is there any real step forward? Indian J Ophthalmol. 2012;60:251-4.

24. Pacella E, Pacella F, La Torre G, Impallara D, Malarska K, Brillante C, et al. Testing the effectiveness of intravitrealRanibizumab during 12 months of follow-up in venous occlusion treatment. Clin Ter. 2012;163:e413-222.

25. Hayreh SS, Zimmernam B, McCarthy MJ, Podhajsky P. Systemic diseases associated with various types of retinal vein occlusion. Am J Ophthalmol. 2001;131:61-77.

26. Shima C, Sakaguchi H, Gomi F,Kamei M, Ikuno Y, Oshima Y et al. Complications in patients after intravitreal injection of bevacizumab. Acta Ophthalmol. 2008;86:372-6.

27. Avery RL. Regression of retinal and iris neovascularisation after intravitreal injection of bevacizumab (avastin) treatment. Retina 2006;26.352-4. 
28. Iturralde D, Spaide RF, Meyerle CB, Klancnik JM, Yannuzzi LA, Fisher YL, et al. Intravitrealbevacizumab (Avastin) treatment of macular edema in central retinal vein occlusion: a short-term study. Retina 2006;26:279-84.

29. Chiquet C, Bron AM, Straub M, Dupuy C, Isaico R, Aptel F, et al. RetinalVein Occlusions: Therapeutic Switch in Macular Oedema Treatment with a 12-Month Follow-Up. Ophthalmic Res. 2016;55:152-8.

30. MayerK, Keese M, Schmandra TC, Meyn M, Schmitz-Rixen T. Diagnosis and therapy of deep vein thrombosis. MMW Fortschr Med. 2012;154:79-83.

31. Hayreh SS. Prevalent misconceptions about acute retinal vascular occlusive disorders. Prog Retin Eye Res. 2005;24:493-519.

32. Rehak M, Wiedemann P. Retinal vein thrombosis: pathogenesis and management. J Thromb Haemost. 2010;8:1886-94.

33. Scott IU, Ip MS, VanVeldhuisen PC, Oden NL, Blodi BA, Fisher M, et al. A randomized trial comparing the efficacy and safety of intravitreal triamcinolone with standard care to treat vision loss associated with macular Edema secondary to branch retinal vein occlusion: the Standard Care vs Corticosteroid for Retinal Vein Occlusion (SCORE) study report 6. Arch Ophthalmol. 2009;127:1115-28.

34. de Smet MD. Corticosteroid intravitreal implants. Dev Ophthalmol. 2012;51:122-33.

35. Gewaily D, Muthuswamy K, Greenberg PB. Intravitreal steroids versus observation for macular edema secondary to central retinal vein occlusion. Cochrane Database Syst Rev. 2015;9:CD007324. 


\section{Figure caption}

Figure 1. Best corrected visual acuity using ETDRS in patients affected by branch (BRVO) and central retinal vein occlusion (CRVO) treated with dexamethasone implant (Ozurdex®) over six months. Data are the mean \pm SEM. Data are expressed in letters (EDTRS values). T0: baseline; T1: 1 month; T3: 3 months; T6: 6 months post injection. Asterisk (*) indicates significant difference between the groups. $* \mathrm{p}<0.05 ; * * \mathrm{p}<0.01$.

Figure 2. Best corrected visual acuity using ETDRS in patients affected by retinal vein occlusion (RVO) and treated with dexamethasone implant within or after 7 days from diagnosis. Data are the mean \pm SEM. Data are expressed in letters (EDTRS values). T0: baseline; T1: 1 month; T3: 3 months; T6: 6 months post injection. $\leq 7 \mathrm{~d}=$ within 7 days from diagnosis; $>7 \mathrm{~d}=$ after 7 days from diagnosis. Asterisk (*) indicates significant difference between the groups. **p<0.01.

Figure 3. Central macular thickness (CMT) in patients affected by branch (BRVO) and central retinal vein occlusion (CRVO) treated with Intravitreal Dexamethasone Implant (Ozurdex®) over six months. Data are the mean \pm SEM. Values are expressed in $\mu$ m.T0: baseline; T1: 1 month; T3: 3 months; T6: 6 months post injection. Asterisk (*) indicates significant difference between the groups. $* \mathrm{p}<0.05 ; * *_{\mathrm{p}}<0.01$.

Figure 4. Central macular thickness (CMT) in patients affected by retinal vein occlusion (RVO) and treated with dexamethasone implant within or after 7 days from diagnosis. Data are the mean \pm SEM. Values are expressed in $\mu \mathrm{m}$. T0: baseline; T1: 1 month; T3: 3 months; T6: 6 months post injection. . $\leq 7 \mathrm{~d}=$ within 7 days from diagnosis; $>7 \mathrm{~d}=$ after 7 days from diagnosis. Asterisk $(*)$ indicates significant difference between the groups. $* * \mathrm{p}<0.01$. 
Figure 1.Early TreatmentDiabetic Retinopathy Study (ETDRS) in patients affected by

BRVO and CRVO treated with dexamethasone implant

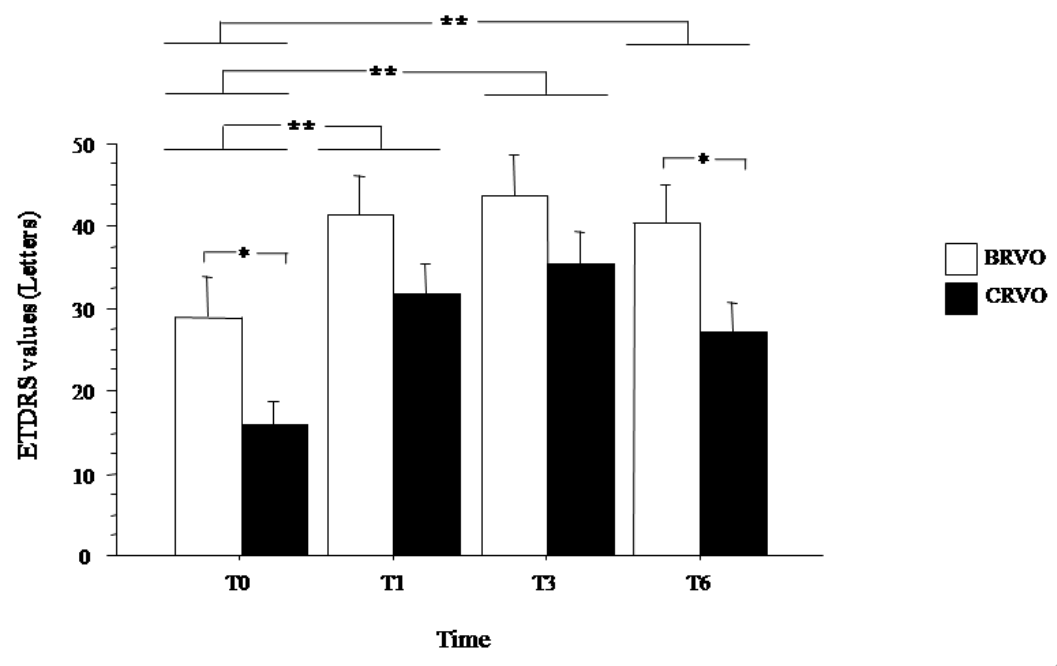

Figure 2.Early TreatmentDiabetic Retinopathy Study (ETDRS) in patients affected by RVO and treated with dexamethasone implant within or after 7 days from diagnosis

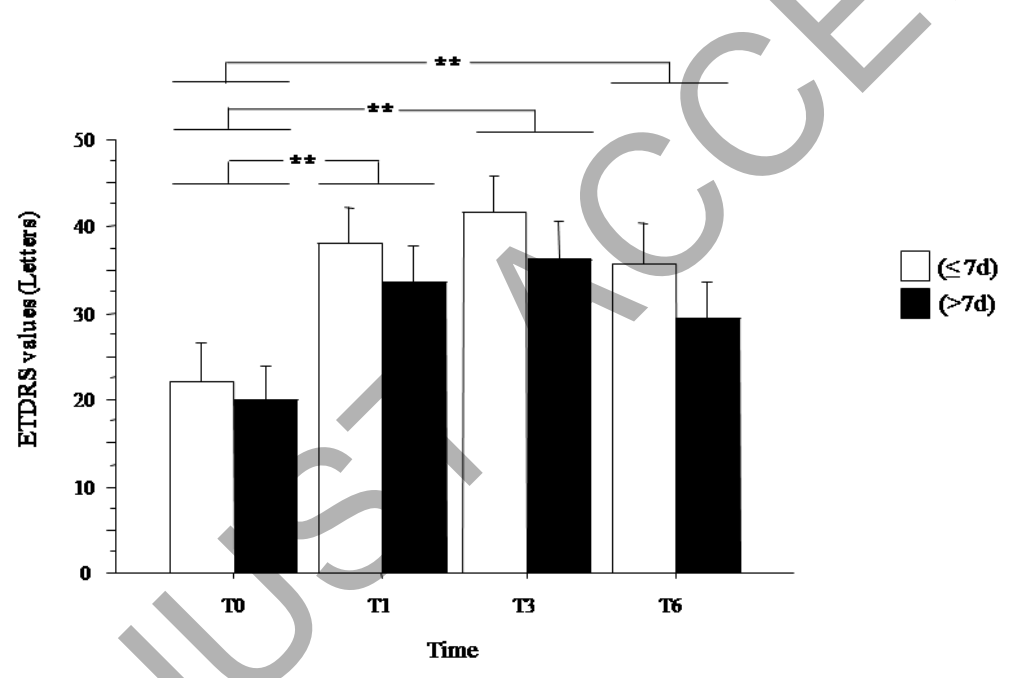


Figure 3. Central macnlar thickness (CMT) in patients affected by BRVO and CRVO treated with dexamethasone implant

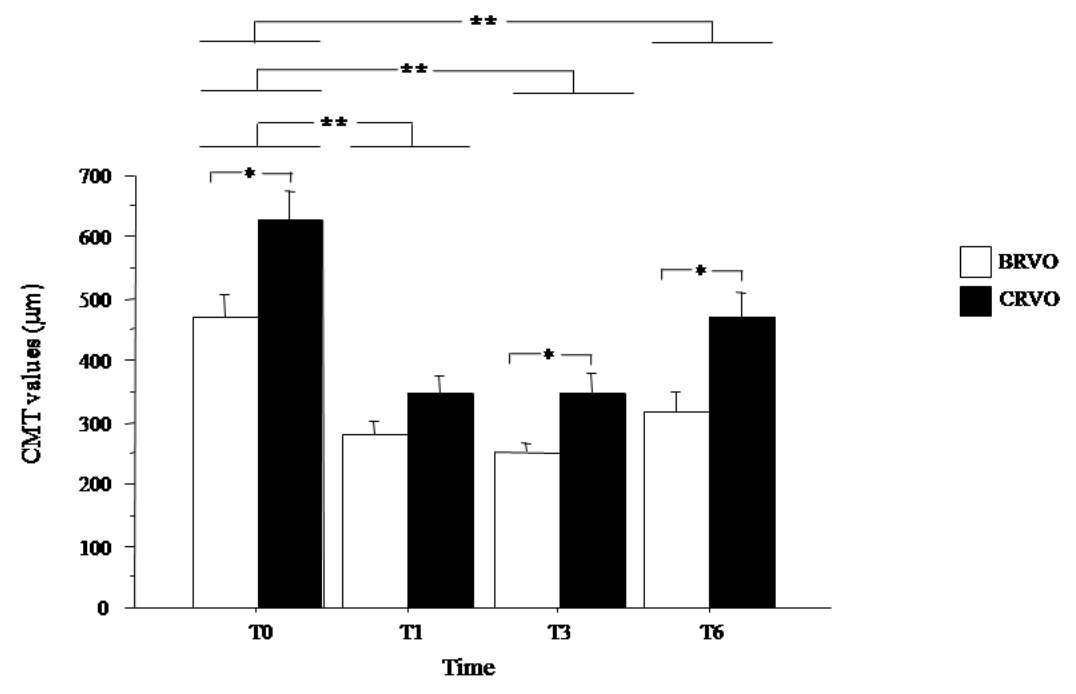

Figure 4. Central macular thickness(CMT) in patients affected by BRVO and CRVO and treated with dexamethasone implant within or after 7 days from diagnosis

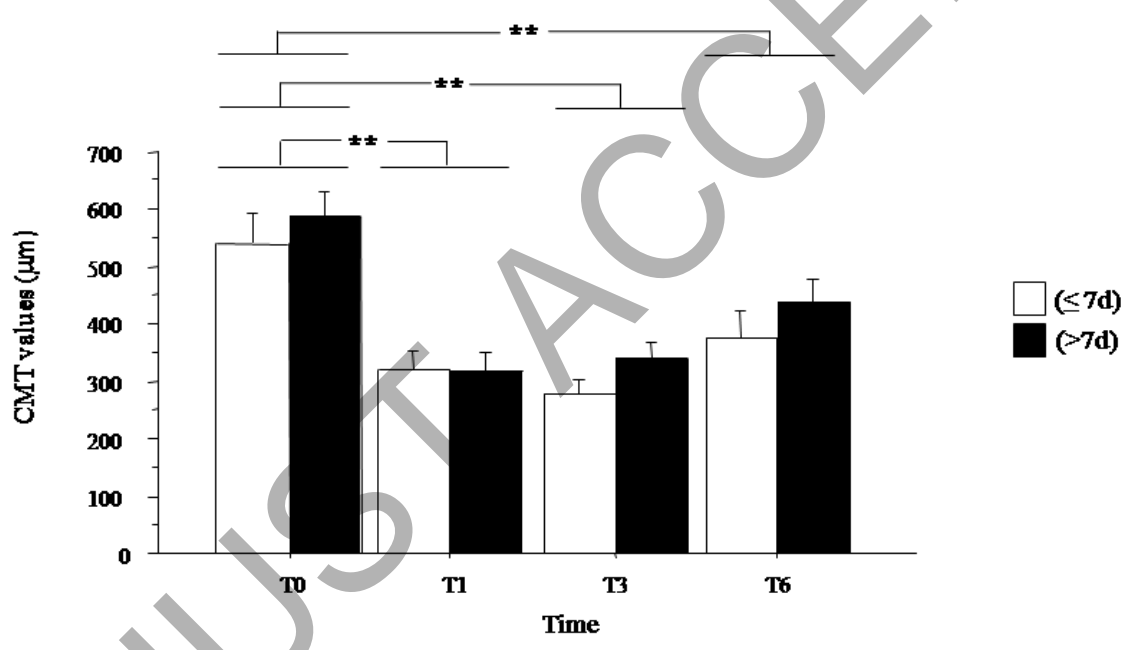


Table 1. Demographic and clinical features of the patients affected by retinal vein occlusion included in the study.

\begin{tabular}{l|c}
\hline Number of patients & 81 \\
\hline Number of treated eyes & 81 \\
\hline Sex & $\mathrm{M}=53 ; \mathrm{F}=28$ \\
\hline Age (years) & $62.5 \pm 15.31$ \\
\hline Number of patients treated $\leq 7$ days & $38(22 \mathrm{BRVO} / 16 \mathrm{CRVO})$ \\
\hline Number of patients treated $>$ 7 days & $43(13 \mathrm{BRVO} / 30 \mathrm{CRVO})$ \\
\hline Number of patients affected by BRVO & 35 \\
\hline Number of patients affected by CRVO & 46 \\
\hline
\end{tabular}

Data are expressed as mean \pm standard deviation. M: male; F: female; branch retinal vein occlusion (BRVO); central retinal vein occlusion (CRVO).

Table 2. Early Treatment Diabetic Retinopathy Study (EDTRS) and Central macular thickness (CMT) values at baseline and in the successive follow-up points.

\begin{tabular}{|c|c|c|c|c|}
\hline \multirow[t]{2}{*}{ Time points } & \multicolumn{2}{|c|}{$\begin{array}{c}\text { Visual Acuity (number of letters } \\
\text { ETDRS) }\end{array}$} & \multicolumn{2}{|c|}{ CMT $(\mu \mathrm{m})$} \\
\hline & BRVO & CRV & BRVO & CRVO \\
\hline Baseline (TO) & $28.94 \pm 20.62$ & $15.76 \pm 15.56$ & $472.00 \pm 143.67$ & $626.96 \pm 246.68$ \\
\hline 1 month $(T 1)$ & $41.35 \pm 19.92$ & $31.80 \pm 18.69$ & $279.94 \pm 88.32$ & $345.23 \pm 163.30$ \\
\hline 3 months (T3) & $43.82 \pm 20.34$ & $35.34 \pm 19.63$ & $253.11 \pm 47.52$ & $346.84 \pm 159.89$ \\
\hline 6 months (T6) & $40.35 \pm 19.82$ & $27.03 \pm 18.84$ & $317.00 \pm 130.36$ & $468.88 \pm 213.67$ \\
\hline
\end{tabular}

Data are the mean \pm standard deviation. BRVO: branch retinal vein occlusion; CRVO: central retinal vein occlusion. 